Send your letters to the Editor,

British Dental Journal,

64 Wimpole Street

London

W1G 8YS

Email bdj@bda.org

Priority will be given to letters less than 500 words long.

Authors must sign the letter, which

may be edited for reasons of space.

\section{DUDLEY BUXTON}

Sir, I am researching the history of the Emergency Medical Service (EMS) Maxillofacial Unit established at Hill End Hospital in St Albans by Rainsford Mowlem and John Lycester Dudley Buxton in 1939 for the treatment of military and civilian war casualties. My reason for writing is that despite being a prominent member of the dental profession, and at one time Sub-Dean of University College Hospital Dental School and honorary surgeon to the Royal Dental Hospital, I am having great difficulty in unearthing any factual information about the life of Dudley Buxton - searches through the British Dental Journal for an obituary, the history of the Royal Dental Hospital, and a partial history of University College Hospital for any reference to him have all drawn a blank. I would be most grateful therefore to hear from any readers who could provide information that would enable a short biographical note to be put together. My address is: Visiting Professor, Faculty of Dentistry, National University of Singapore, 5 Lower Kent Ridge Road, Singapore 119074; email pndmcm@nus.edu.sg.

\section{C. Meikle \\ Singapore \\ DOI: 10.1038/sj.bdj.2009.909}

\section{NEGATIVE TERMINOLOGY}

Sir, a referral letter from a general dental practitioner to a hospital consultant was recently viewed, describing a patient as 'deaf and dumb.' What is worse, the reply contained the same expression. Terms such as 'deaf and dumb' or 'deafmute' should certainly be avoided. The term 'dumb' in modern language tends to denote 'stupidity' or 'idiocy.' The term 'mute' refers to an individual who cannot produce speech and 'mutism' is a medical or psychological condition, ie the inability or refusal to produce sounds, respectively. The majority of deaf individuals have normal vocal chords but prefer to communicate via sign language, as it is difficult to modulate the voice without hearing. Derogatory terminology such as 'deaf and dumb' has been found to be uncommon in the international English language press. ${ }^{1}$ Such terms should, hopefully, fade from acceptable usage and medical practitioners may help lead the way.

On 25 November 1845, the novelist, playwright and human rights activist Victor Hugo (1802-1885) wrote to Ferdinand Berthier, a prominent deaf man in Paris: 'What matters deafness of the ear, when the mind hears. The one true deafness, the incurable deafness, is that of the mind.'

The term 'deaf' refers to a physical condition characterised by a severe or total lack of auditory sensitivity to sound. Deafness may be categorised by the degree of hearing loss, which may include:

- slight hearing loss (16-25 dB)

- mild hearing loss (26-40 dB)

- moderate hearing loss (41-55 dB),

- moderately severe hearing loss (56-70 dB)

- severe hearing loss (71-90 dB),

- profound hearing loss (91-120 dB)

- or total deafness (120 dB or more of hearing loss).

It is incumbent upon all medical practitioners to use language that is not derogratory, negative or offensive to anyone, particularly patients.

F. B. Naini By email
1. Power D. Googling 'deaf': deafness in the world's English-language press. Am Ann Deaf 2006-2007; 151: 513-518.

2. Grant E M. The career of Victor Hugo. Cambridge, MA: Harvard University Press, 1946.

DOI: 10.1038/sj.bdj.2009.910

\section{PROTOCOL REQUIRED}

Sir, it is with great interest and appreciation I read the letter to the editor written by J. Gollings published in the $B D J$ (2009; 207: 53) titled GPs and bisphosphonates. I would like to make a comment in support of this letter.

I am working in the community dental services and a major proportion of our patients are elderly and on multiple drug therapy. General practitioners who provide medical care for these patients seem to be unaware of the dental implications of these medications. One group of medications is 'bisphosphonates' for which every dental centre in the UK has their own policies and protocols and of which the medical fraternity seem to be ignorant. No referral is made even if these patients have a mouth full of decayed teeth which might need extraction sooner or later.

I suggest there should be a written protocol for dental referral for all patients before they start a course of bisphosphonate therapy, as exists for patients who have to undergo radiotherapy or chemotherapy.

Z. Imran

Dundee

DOI: 10.1038/sj.bdj.2009.911

\section{DENTAL CARE ARTISTS}

Sir, your leader of hearts and heads (BDJ 2009; 207: 605) expresses one of the key elements of what it takes to be a primary care practitioner. The technical rational world expressed by the 'science' of dentistry has to be applied to what Donald Schön describes as the "swampy 
lowlands' of professional practice. ${ }^{1}$ This application of science to the patient in our care is the 'art' of dentistry.

Had I had the good fortune to be at the Glasgow conference, I would have liked to challenge Charan Gill on his stated reliance on 'gut instinct'. Like you, I have real concerns about dentists being inspired to run their professional lives by gut instinct - whether on the clinical or business side of primary care.

I believe that Charan's apparently intuitive decision-making is actually based on some fairly objective and very immediate feedback. His years of experience and reflection have probably created a tacit knowledge of his business, but I would contend his decisions are not based on a whim. This matches the unconscious knowledge that Schön observed as the 'art' of professional practice.

Charan has a couple of factors in his favour in comparison to primary dental care. I would have thought that the success or failure of one of his new ventures (the equivalent of one of our treatment plans?) would be evident very quickly by a number of statistically sound indicators (such as profit/loss or footfall). This direct and accurate feedback means success for Charan can be measured within months. Our treatment plans may take years to demonstrate their effectiveness. And, as you point out, Charan's failure is only a risk for the investor.

Schön found that professional artistry is not something ethereal. We can learn how to balance this heart and head decision making, to be better primary dental care artists. There are a number of ways of achieving this, of which I offer just three.

Firstly, when applying the technical rational 'evidence' of dentistry, we have to understand that no research finding can be applied directly to the problem in front of us. The best we can hope to achieve is practice that is evidence informed. This requires us to develop an evidence base that is as closely related to the practice setting as possible. Ideally, this research should be performed in practice with the direct involvement of the dental team. Evidence derived from the bench will require translational research to ensure its findings are effectively applied.
The relevant reporting of evidence is also key, and I commend the excellent Evidence Based Dentistry journal for pointing readers to the significance of findings to practice. The FGDP journal, Primary Dental Care, is the only practice-based research publication in the world and will become an increasingly valuable source.

Finally, teaching and assessment of the primary care team must develop their skills of artistry in applying the evidence, not just their ability to remember technical rational facts. I would encourage practitioners to seek out courses that teach them to critically appraise the evidence, with exams that test their application; and, for the hardy few, directly involve themselves in practice-based research.

In dentistry's current 'modern world', an entrepreneurial approach to services is clearly being encouraged by many of its leaders, from the BDA to the Department of Health. In the dissolution of old attitudes and systems, the dental profession is becoming very creative in their activities - sometimes bizarrely so. Personally, I am strongly in favour of creating new and better solutions to our many challenges. However, unlike Charan, we also have a professional responsibility to the public to ensure we gather, learn and apply the best available evidence. Ethically, we cannot rely on gut instinct. We have to exercise the skill of professional artistry; to balance the heart and head.

1. Schön D. The reflective practitioner. London: Temple Smith, 1983.

A. Toy

Nottingham

DOI: 10.1038/sj.bdj.2009.912

\section{RATIO TASTE}

Sir, I thank Mr S. Radlinskiy for his kind words about my work on the golden proportion and his contribution to the discussion regarding proportion in dental aesthetics (Aesthetic deviation; BDJ 2009; 206: 447).

I would agree with him that the sizes of incisors are usually the same width both sides and any differences in symmetry are due to their positioning in the dental arch and I like his phrase that 'Ultimately mathematics is the root of aesthetics.' Some further elaboration on this profound statement would be welcome.
I do, however, wonder where he gets his idea from there that the optimum aesthetic ratio between the upper incisors should be $1.3: 1.0: 1.15$.

Is this his own decision and his own taste, or is it in the literature, or does he base it on anything other than his own opinion? Thank you.

E. Levin

By email

DOI: 10.1038/sj.bdj.2009.913

\section{TIME TO RETIRE}

Sir, I recently saw this new patient who came complaining of food packing between the lower left first molar and the implant retained crown in the lower left second molar space (Figs 1-2). I think I have now seen everything and it is time for me to retire!

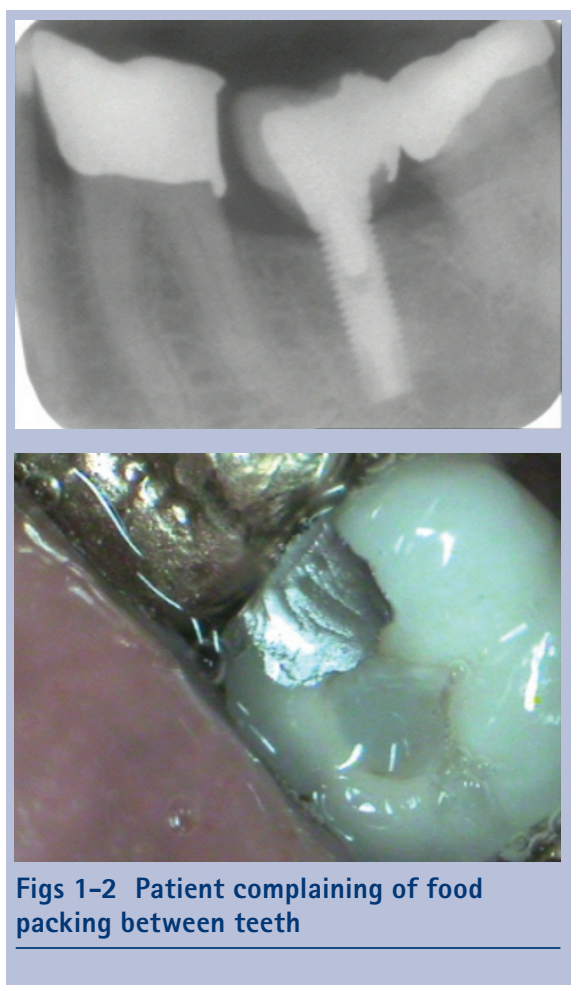

\section{J. G. McLaughlin Newton Abbot DOI: 10.1038/sj.bdj.2009.914}

\section{STRAY TEETH}

Sir, despite many years of experience in oral surgery, occasionally I come across a situation that keeps me on my toes. An apparently routine case may not always be predictable and can take one by surprise. The following case is an example of this and relates how the problem posed was eventually resolved 
by the combined approach of surgeon and anaesthetist.

A 13-year-old girl was admitted to hospital as a day case patient for surgical removal of a palatally-impacted, unerupted upper right second premolar together with extraction of the upper left second premolar and both lower first premolars under general anaesthesia administered by a consultant anaesthetist.

Following anaesthetic induction she was intubated with a cuffed nasal tube via her right nasal airway and a gauze throat pack was inserted into the inferior region of the oropharynx. In order to optimise surgical access to the palate, the top end of the operating table was dropped to allow for neck extension and in addition the table was adjusted to give some head-down tilt.

Following local anaesthetic infiltration a palatal mucoperiosteal flap was raised to reveal the crown of the upper right second premolar. This tooth was very simply elevated but unfortunately dropped into the oropharynx. Suction of the oropharynx, into which some blood was accumulating, was immediately carried out but the tooth could not be located.

Due to the position of the patient, with her nasopharynx being lower than her oropharynx, it was suspected that the tooth may have passed into the nasopharyngeal region. Careful suction of the accessible region behind the soft palate was carried out but this failed to retrieve the tooth. At this point the palatal flap was sutured in order to achieve haemostasis and optimise visualisation of the pharynx. A laryngoscope was inserted into the oropharynx in order to visualise the throat pack and to confirm that the tooth had not fallen onto its surface. A fibre-optic scope was then passed behind the soft palate in order to locate the position of the tooth in the nasopharynx. The patient's position was then adjusted to use gravitation force. The neck was straightened and the table adjusted for head-up tilt but this did not deliver the tooth into the oropharynx. Further gentle mobilisation of the patient's head together with flushing of water into the left nasal airway similarly failed to dislodge the tooth. The tube was then replaced with a laryngeal mask. Before this was carried out the patient's position was again adjusted to give maximum head-down tilt in order to ensure that the tooth remained in the nasopharynx whilst the tube was changed.

After removal of the nasal tube, placement of a laryngeal mask, replacement of the throat pack and further repositioning of the patient to a head-up tilt, the tooth remained elusive. However, following flushing of the right nasal passage with water, whilst occluding the left nasal airway to achieve maximum flushing pressure, the tooth finally appeared in the oropharynx and was retrieved using Fickling forceps. The tooth was found to have a somewhat shortened root which may have contributed to the ease of its elevation and subsequent loss and also to its ease of passage in a cranial direction in the nasopharynx.

In order to avoid this problem occurring again, when patients are positioned with a head-down tilt I will, in addition to the anaesthetist's pack, be placing a further pack in the accessible oropharynx as a safety net to capture any stray teeth!

\section{J. K. A. Parker} Hereford DOI: 10.1038/sj.bdj.2009.915

\section{GUARANTEES FOR DENTAL WORK}

Sir, I note Professor Steele's proposal that dental treatment should carry a three year guarantee. Is that all dental treatment or just that provided on the NHS?

Many years ago a British dentist working in California explained in an article how they also had a requirement to guarantee their work. This they were happy to do. However, the flip side was that the patient had to guarantee, in writing, to look after the work $100 \%$ once it was in place. The presence of any plaque in the mouth negated the dentists' guarantee.

Can we assume the same requirements in the UK?

A. Caen By email DOI: 10.1038/sj.bdj.2009.916 